\title{
Prediction of Insulin Resistance and Impaired Fasting Glucose Based on Sex Hormone-Binding Globulin (SHBG) Levels in Polycystic Ovary Syndrome
}

\author{
Aleksandra Biernacka-Bartnik, ${ }^{1}$ Piotr Kocełak ${ }^{(D)}{ }^{2}$ Aleksander Jerzy Owczarek ${ }^{(D},{ }^{3}$ \\ Piotr Choręza $\left(\mathbb{D},{ }^{4}\right.$ Monika Puzianowska-Kuźnicka $\mathbb{D}^{5,6}$ Leszek Markuszewski ${ }^{5},{ }^{7}$ \\ Paweł Madej $\mathbb{D}$, ${ }^{1}$ Jerzy Chudek $\mathbb{D}^{2,8}$ and Magdalena Olszanecka-Glinianowicz $\mathbb{D}^{3}$ \\ ${ }^{1}$ Department of Gynecological Endocrinology, Faculty of Medical Sciences in Katowice, Medical University of Silesia in Katowice, \\ Katowice, Poland \\ ${ }^{2}$ Pathophysiology Unit, Department of Pathophysiology, Faculty of Medical Sciences in Katowice, \\ Medical University of Silesia in Katowice, Katowice, Poland \\ ${ }^{3}$ Health Promotion and Obesity Management Unit, Department of Pathophysiology, Faculty of Medical Sciences in Katowice, \\ Medical University of Silesia in Katowice, Katowice, Poland \\ ${ }^{4}$ Department of Statistics, Department of Instrumental Analysis, Faculty of Pharmaceutical Sciences in Sosnowiec, \\ Medical University of Silesia, Katowice, Poland \\ ${ }^{5}$ Department of Human Epigenetics, Mossakowski Medical Research Institute, Polish Academy of Sciences, Warsaw, Poland \\ ${ }^{6}$ Department of Geriatrics and Gerontology, Medical Centre of Postgraduate Education, Warsaw, Poland \\ ${ }^{7}$ Center Heart and Vascular Diseases Internal and Metabolic Diseases Mazovian Specialist Hospital in Radom, \\ University of Humanities and Technology in Radom, Radom, Poland \\ ${ }^{8}$ Department of Internal Medicine and Oncological Chemotherapy, Faculty of Medical Sciences in Katowice, \\ Medical University of Silesia in Katowice, Katowice, Poland
}

Correspondence should be addressed to Magdalena Olszanecka-Glinianowicz; magolsza@gmail.com

Received 20 July 2021; Revised 15 September 2021; Accepted 8 December 2021; Published 31 January 2022

Academic Editor: Janaka Karalliedde

Copyright (c) 2022 Aleksandra Biernacka-Bartnik et al. This is an open access article distributed under the Creative Commons Attribution License, which permits unrestricted use, distribution, and reproduction in any medium, provided the original work is properly cited.

Objective. Decreased synthesis of sex hormone-binding globulin (SHBG) related to hyperinsulinemia is one of the disturbances characteristic of polycystic ovary syndrome (PCOS). Hyperinsulinemia is a compensatory mechanism for liver insulin resistance (IR); thus, SHBG may be considered as a surrogate marker of liver IR. Therefore, this study aimed to assess the prediction of IR and impaired fasting glucose (IFG) based on SHBG levels in women with PCOS. Methods. This analysis included data retrieved from medical records of 854 patients with PCOS hospitalized in the Gynecological Endocrinology Clinic from 2012 to 2019. Data including anthropometric parameters, fasting plasma glucose, insulin, and SHBG levels were analyzed. BMI and HOMA-IR were calculated with standard formulas. Results. IFG and IR assessed based on HOMA-IR values $>2.0$ were found in 19.5\% and 47.8\% of the study group, respectively. Empirical optimal cutoff values for SHBG levels were $\leq 41.5 \mathrm{nmol} / \mathrm{L}$ typical for IR (AUC 0.711, sensitivity $61.1 \%$, specificity $71.6 \%$, positive predictive value (PPV) $70.7 \%$, and negative predictive value (NPV) $62.1 \%$ ). The probability of insulin resistance occurrence for SHBG concentration $26.1 \mathrm{nmol} / \mathrm{L}$ (the lower normal range) was $61.6 \%$ (95\% CI: $57.4 \%-65.8 \%$ ). The SHBG concentration of $36.4 \mathrm{nmol} / \mathrm{L}$ and $8.1 \mathrm{nmol} / \mathrm{L}$ was related to a $10 \%$ and $20 \%$ probability of IFG, respectively. Conclusion. In conclusion, this is the first study estimating the probability of liver IR and IFG occurrence based on SHBG levels in women with PCOS. Despite the low sensitivity, SHBG level below $42 \mathrm{nmol} / \mathrm{L}$ should cause closer monitoring for the fatty liver and prediabetes. 


\section{Introduction}

According to some recent studies, obesity, especially visceral obesity, is an important factor contributing to hormonal and metabolic disturbances in polycystic ovary syndrome (PCOS) [1,2]. Increased volume of adipocytes causes an influx of macrophages into visceral adipose tissue and stimulates the production of proinflammatory cytokines. Emerging low-grade chronic inflammation induces hormonal disturbances of adipose tissue and insulin resistance (IR) initially locally and then in the liver and muscles $[3,4]$. The increased insulin level, a compensatory mechanism for liver and insulin resistance, as well as the decreased circulating SHBG level are typical for PCOS. Notably, hyperinsulinemia is one of the factors inhibiting SHBG synthesis in the liver. Therefore, SHBG may be considered as a surrogate marker of hepatic insulin resistance [5-7].

The occurrence of impaired glucose tolerance (IGT) in women with PCOS is estimated at $31-35 \%$ while in the general population of women of reproductive age at $1.6 \%$. Similarly, type 2 diabetes occurs in $7.5-10 \%$ and $2.2 \%$ of women, respectively [8-10]. It has been shown that the 2year risk of developing prediabetes and type 2 diabetes in PCOS women with normal glucose tolerance is 2.5-fold higher than in women without this syndrome [11].

A retrospective analysis of a large primary-care cohort of 63,120 women with PCOS and 121,064 women without this syndrome revealed a more frequent occurrence of nonalcoholic fatty liver disease (NAFLD) among PCOS women. Moreover, $\mathrm{SHBG}<30 \mathrm{nmol} / \mathrm{L}$ and testosterone $>3 \mathrm{nmol} / \mathrm{L}$ were associated with a higher incidence of NAFLD [12]. The association between lower SHBG levels and the occurrence of NAFLD was confirmed in a meta-analysis of 16 studies including 13,721 men and 5,840 women. It should be noted that this relationship was stronger in women than in men [13]. Enhanced gluconeogenesis resulting in impaired fasting glucose (IFG) is typical for NAFLD. In a cohort of 2,654 men, lower SHBG levels were associated with prediabetes status [14]. Moreover, in the 5-year follow-up of 2,077 women, SHBG concentrations were inversely associated with the risk of development of type 2 diabetes [15]. Furthermore, another 5-year follow-up study (Environment, Inflammation, and Metabolic Diseases Study) showed that low SHBG levels were associated with 4-fold higher risk of developing type 2 diabetes both in men and women. The risk was decreasing with the increase in SHBG tertile after multifactorial adjustment in women [16]. The association between low SHBG levels and increased risk of development of type 2 diabetes was supported by the Rotterdam study including 3,177 postmenopausal women, with a median observation of 11.1 years [17]. Also, a 6-year follow-up study that included 1,377 subjects showed that SHBG level is a marker of insulin resistance development [18]. O'Reilly et al. [19] have shown that the increased risk of developing type 2 diabetes occurred in men with SHBG $<40 \mathrm{nmol} / \mathrm{L}$ and in women with SHBG $<50 \mathrm{nmol} / \mathrm{L}$. Some other studies found low SHBG levels being the risk factor for gestational diabetes [20-23]. Hedderson et al. [21] observed that SHBG levels below the median $(<64.5 \mathrm{nmol} / \mathrm{L})$ and $\mathrm{BMI}>25 \mathrm{~kg} / \mathrm{m}^{2}$ were associated with a 5-fold increase in the risk of development of gestational diabetes. On the other hand, in a study of 180 pregnant women with PCOS, an increase in SHBG concentration by $1 \mathrm{nmol} / \mathrm{L}$ reduced the risk of developing gestational diabetes by $7 \%$ [22]. Finally, the results of a metaanalysis of 26 studies showed that SHBG testing in early pregnancy may be a marker of the risk of developing gestational diabetes [23].

Based on the abovedescribed studies, we hypothesized that SHBG levels may serve as a surrogate marker of hepatic insulin resistance in PCOS women. Therefore, this study aimed to assess the value of prediction of insulin resistance and impaired fasting glucose based on SHBG level in women with PCOS.

\section{Materials and Methods}

This retrospective study includes data from the medical records of 854 Caucasian female patients of the Gynecological Endocrinology Clinic for the first time diagnosed with PCOS based on the Rotterdam criteria during index hospitalization that took place between 2012 and 2019 .

The inclusion criteria comprised age $18-30$ years and diagnosis of PCOS. The exclusion criteria were diagnosis of type 2 diabetes and other endocrinological disturbances, any currently ongoing pharmacological therapy and past bariatric treatment of obesity, and the lack of completeness of necessary datasets in the medical records.

The analyzed dataset included age, body mass, height, and routine measurements of fasting glucose, insulin, and SHBG levels, all performed in a single hospital laboratory using the same set of methods for all study subjects. Glucose concentration was measured using the colorimetric method (Roche Diagnostic GmbH, Mannheim, Germany). Insulin and SHBG levels were determined using the ECLIA method (Roche, reagents for Cobas E411). BMI and HOMA-IR values were calculated with standard formulas.

The retrospective analysis did not fulfill the criteria of a medical experiment and, therefore, did not require the consent of a bioethical committee and the patient. Personal data were not proceeded in the analysis.

2.1. Data Analysis. The study group was divided according to an HOMA-IR cutoff value of 2.0 [24] into the subgroups with $(N=408 ; 47.8 \%)$ and without insulin resistance $(N=446 ; 52.2 \%)$.

2.2. Statistical Analysis. Statistical analysis was performed using STATISTICA 13.0 PL (TIBCO Software Inc., Palo Alto, CA, U.S.), StataSE 13.0 (StataCorp LP, TX, U.S.), and R software (R Core Team (2013). R: a language and environment for statistical computing, $\mathrm{R}$ Foundation for Statistical Computing, Vienna, Austria, URL http://www. R-project.org/. Statistical significance was set at a $p$ value below 0.05 . All tests were two tailed. Imputations were not performed for missing data. Nominal and ordinal data were expressed as percentages. Interval data were expressed as mean \pm standard deviation in case of normal 
data distribution or as median (lower-upper quartiles) in data with nonnormal or skewed distribution. The distribution of variables was evaluated by the Shapiro-Wilk W test and the quantile-quantile (Q-Q) plot. To compare two groups with HOMA $\geq 2.0$ and HOMA $<2.0$, the Student's $t$-test for independent data or the $U$ Mann-Whitney test was used, according to data distribution, and homogeneity of variances was assessed by the Fisher-Snedecor F test. To find a cutoff point discriminating the insulin resistance and impaired fasting glucose based on the SHBG level, parametric and nonparametric ROC curves were calculated with the area under curve (AUC) and corresponding sensitivity, specificity, and positive and negative predictive values. The fractional polynomial curve with a $95 \%$ confidence interval was calculated to show the associations between SHBG levels and insulin levels. The logistic regression was used to assess the probability of IR and IFG based on the SHBG levels.

\section{Results}

Insulin resistance assessed based on HOMA-IR values 2.0 and above was found in $47.8 \%(N=408)$ of the study group and IFG in $9.4 \%(N=80)$. Compared to patients without IR, the IR group was characterized by significantly higher BMI values and more frequent occurrence of obesity and significantly higher fasting glucose and insulin concentrations, as well as more common IFG, lower median SHBG levels (30.8 vs. $49.1 \mathrm{nmol} / \mathrm{L} ; p<0.001)$, and a more frequent occurrence of SHBG concentration below laboratory's lower limit of the normal range for women aged 18-50 years $(<26.1 \mathrm{nmol} / \mathrm{L})$. The detailed study groups characteristics are listed in Table 1.

As expected, the median SHBG level was the lowest in the subgroup with obesity and the highest in the normal weight subgroup (Table 2 and Figure 1). On the contrary, glucose and insulin levels were the highest in the subgroup with obesity and the lowest in the normal weight subgroup. It addition, the percentage of women with insulin resistance and with SHBG level below the lower limit of the reference range $(<26.1 \mathrm{nmol} / \mathrm{L})$ was the highest in the subgroup with obesity and the lowest in the normal weight subgroup (Table 2).

The median SHBG levels were significantly higher in patients with normal insulin levels than in these with levels above laboratory's reference range $(>25 \mu \mathrm{IU} / \mathrm{mL})$ $(36.9 \mathrm{nmol} / \mathrm{L}$ (24.4-53.8) vs. $25.3 \mathrm{nmol} / \mathrm{L}$ (17.6-39.5); $p<0.01$ ) (Figure 2). Empirical optimal cutoff values for SHBG levels, based on the ROC analysis, that characterize individuals with IR were $\leq 41.5 \mathrm{nmol} / \mathrm{L}$ (AUC 0.71 , sensitivity $61.1 \%$, specificity $71.6 \%$, positive predictive value (PPV) $70.7 \%$, and negative predictive value (NPV) 62.1\%), Table 3.

We observed $10 \%$ probability of insulin resistance occurrence at an SHBG concentration of $111.1 \mathrm{nmol} / \mathrm{L}, 20 \%$ at $85.3 \mathrm{nmol} / \mathrm{L}, 25 \%$ at $76.1 \mathrm{nmol} / \mathrm{L}, 33.3 \%$ at $63.2 \mathrm{nmol} / \mathrm{L}$, and $50 \%$ at $41.1 \mathrm{nmol} / \mathrm{L}$, while the probability of IR for an SHBG concentration of $26.1 \mathrm{nmol} / \mathrm{L}$ was $61.6 \%$ (95\% CI: 57.4-65.8). Also, there was a $10 \%$ probability of IFG occurrence at an SHBG concentration $36.4 \mathrm{nmol} / \mathrm{L}$ and $20 \%$ at $8.1 \mathrm{nmol} / \mathrm{L}$.
The probability of IR at SHBG concentration $36.4 \mathrm{nmol} / \mathrm{L}$ was $53.7 \%$ (95\% CI: $50.0-57.4)$ and at $8.1 \mathrm{nmol} / \mathrm{L} 73.8 \%$ (95\% CI: 68.7-79.1) (Figure 3).

\section{Discussion}

To the best of our knowledge, this is the first study that estimates the probability of insulin resistance and impaired fasting glucose occurrence in women with PCOS according to cutoff values for SHBG levels.

Low circulating SHBG levels are associated with the development of type 2 and gestational diabetes [14-19, 25, 26]. The results of a 15-year follow-up study suggested that a decreased synthesis of SHBG in the liver is an early response to the increased insulin levels corresponding to IR in visceral fat [27]. The mechanism of SHBG synthesis inhibition was linked with leptin [28, 29].

The HOMA-IR is widely used for the assessment of insulin resistance better reflecting hepatic than muscle insulin resistance [30]. In our study, the cutoff HOMA-IR value for diagnosis of insulin resistance was set at 2.0. As expected, SHBG levels were significantly lower in the insulin-resistant patients, and in this group, SHBG levels below the laboratory's reference range for women aged $18-50$ years $(<26.1 \mathrm{nmol} / \mathrm{L})$ and IFG were more frequently present than in patients without IR.

We observed a $50 \%$ probability of IR occurrence at an SHBG concentration of $41.1 \mathrm{nmol} / \mathrm{L}$. It is consistent with the results of the study enrolling 42,034 women in whom the risk of development of type 2 diabetes increased by $299 \%$ when SHBG levels were in the range from 40 to $49.99 \mathrm{nmol} / \mathrm{L}$ [19] and the study showing that the risk of gestational diabetes development increased five-fold with median SHBG levels below $64.5 \mathrm{nmol} / \mathrm{L}$ [21]. However, an SHBG concentration of $111.1 \mathrm{nmol} / \mathrm{L}$ was already associated with a $10 \%$ probability of IR. In our study, based on the ROC analysis, the empirical optimal SHBG cutoff point for insulin resistance was $41.5 \mathrm{nmol} / \mathrm{L}$. This SHBG cutoff point is characterized by lower sensitivity yet higher specificity, with higher positive than negative predictive value. In addition, AUC illustrating the diagnostic power of the test of 0.711 was lower than accepted for most diagnostic tests in the range of 0.80-0.95. Therefore, one should be cautious about the assumption that SHBG concentrations may replace other markers for assessing insulin resistance. However, the results of our study indicate that SHBG concentrations $<42 \mathrm{nmol} / \mathrm{L}$, higher than the values adopted as the lower limit of normal laboratory range $<26.1 \mathrm{nmol} / \mathrm{L}$, should lead to diagnostics for impaired fasting glucose (if it has not already been carried out), as a group of young PCOS women are already at the increased risk of their occurrence. On the other hand, an SHBG concentration of $36.4 \mathrm{nmol} / \mathrm{L}$ was associated with $53.7 \%$ probability of IR and a low, $10 \%$ probability of IFG. However, in our cohort, fasting glucose measurement was performed only once and the already existing IFG may have been overlooked, causing such a large discrepancy. This hypothesis is supported by results obtained by Jaygobal et al. [31] who assessed the biological variability of fasting glucose, insulin, and SHBG in 10 samples obtained every 4 days from 
TABLE 1: The study group characteristics.

\begin{tabular}{|c|c|c|c|}
\hline & $\mathrm{HOMA}<2.0, N=446(52.2 \%)$ & $\mathrm{HOMA}>2.0, N=408(47.8 \%)$ & $p$ \\
\hline Age (years) & $26 \pm 5$ & $26 \pm 6$ & 0.91 \\
\hline BMI $\left(\mathrm{kg} / \mathrm{m}^{2}\right)$ & $23.0 \pm 4.4$ & $30.5 \pm 7.9$ & $<0.001$ \\
\hline Overweight $(N ; \%)$ & $68 ; 15.2$ & $81 ; 19.8$ & 0.08 \\
\hline Obesity $(N ; \%)$ & $35 ; 7.8$ & $211 ; 51.7$ & $<0.001$ \\
\hline Glucose $(\mathrm{mg} / \mathrm{dL})$ & $86.0(81.0-90.0)$ & $91.0(86.0-97.0)$ & $<0.001$ \\
\hline Impaired fasting glucose $(N ; \%)$ & $4 ; 0.9$ & $76 ; 18.6$ & $<0.001$ \\
\hline Insulin $(\mu \mathrm{IU} / \mathrm{mL})$ & $6.1(4.7-7.6)$ & $13.3(10.9-18.2)$ & $<0.001$ \\
\hline SHBG (nmol/L) & $49.1(34.1-68.2)$ & $30.8(20.7-44.5)$ & $<0.001$ \\
\hline SHBG $<26.1(\mathrm{nmol} / \mathrm{L})(N ; \%)$ & $55 ; 12.3$ & $160 ; 39.2$ & $<0.001$ \\
\hline OR impaired fasting glucose & Ref. & $25.3(9.1-69.9)$ & $<0.001$ \\
\hline OR SHBG < $26.1(\mathrm{nmol} / \mathrm{L})$ & Ref. & $4.56(3.23-6.45)$ & $<0.001$ \\
\hline
\end{tabular}

Mean \pm standard deviation or median (lower quartile-upper quartile) or $95 \%$ confidence interval for OR.

TABLE 2: The study subgroups stratified according the BMI categories.

\begin{tabular}{lccc}
\hline & Normal weight, $N=459(53.8 \%)$ & Overweight, $N=149(17.4 \%)$ & Obesity, $N=246(20.8 \%)$ \\
\hline BMI $\left(\mathrm{kg} / \mathrm{m}^{2}\right)$ & $21.1(19.7-22.8)$ & $27.5(26.3-28.7)$ & $34.8(32.0-39.8)$ \\
Age $($ years) & $25(21-29)$ & $25(22-28)$ & $26(22-31)$ \\
Glucose $(\mathrm{mg} / \mathrm{dL})$ & $87(82-91)$ & $88(83-93)$ & $91(86-97)$ \\
IFG $(N ; \%)$ & $20 ; 4.4$ & $12 ; 8.1$ & $48 ; 19.5$ \\
Insulin $(\mathrm{uIU} / \mathrm{mL})$ & $6.7(4.9-9.2)$ & $9.5(7.4-12.2)$ & $<.28$ \\
HOMA-IR & $1.4(1.0-2.0)$ & $2.1(1.5-2.6)$ & $<0.001$ \\
HOMA-IR $\geq 2(N ; \%)$ & $116 ; 25.3$ & $81 ; 54.4$ & $<.001$ \\
SHBG $(\mathrm{nmol} / \mathrm{L})$ & $51.8(36.6-70.7)$ & $34.2(24.0-46.8)$ & $21.2-20.8)$ \\
SHBG $<26.1(N ; \%)$ & $43 ; 9.4$ & $45 ; 30.4$ & $<0.001$ \\
\hline
\end{tabular}

Median (lower quartile-upper quartile).

the same individuals. This study showed that glucose and insulin levels present significant variability which, in addition, was much higher than the variability of SHBG levels. In addition, the results of a meta-analysis of 28 studies conducted in women with and without PCOS $(n=741$ and $n=1224$, respectively) showed that a 10 unit higher BMI reduces insulin sensitivity as assessed by euglycemichyperinsulinemic clamp by $28 \%$ and $15 \%$, respectively, and low SHBG levels are independently associated with a decrease in insulin sensitivity [32]. The authors of this metaanalysis indicate the need to search cutoff points for SHBG levels to diagnose IR, which was the subject of our study.

IFG is a clinical symptom of liver IR. However, it is not known how early it occurs; therefore, SHBG levels may be considered as an early marker of liver IR preceding IFG. It should be also noted that the SHBG cutoff point estimated empirically in our study is an intermediate value between the cutoff point for SHBG levels associated with the risk of fatty liver in PCOS women (below $30 \mathrm{nmol} / \mathrm{L}$ ) [12] and associated with the increased risk of type 2 diabetes development (below $50 \mathrm{mmol} / \mathrm{L}$ ) [19]. Thus, despite the low AUC and sensitivity, the cutoff point established by us may have a clinical significance in the screening towards IR in PCOS women. Our study suggests that the given SHBG levels should initiate diagnostics and treatment of fatty liver to prevent the development of prediabetes. As mentioned above, fatty liver and liver IR result in increased gluconeogenesis and impaired fasting glucose levels. However, it should be noted that low SHBG is not an established diagnostic

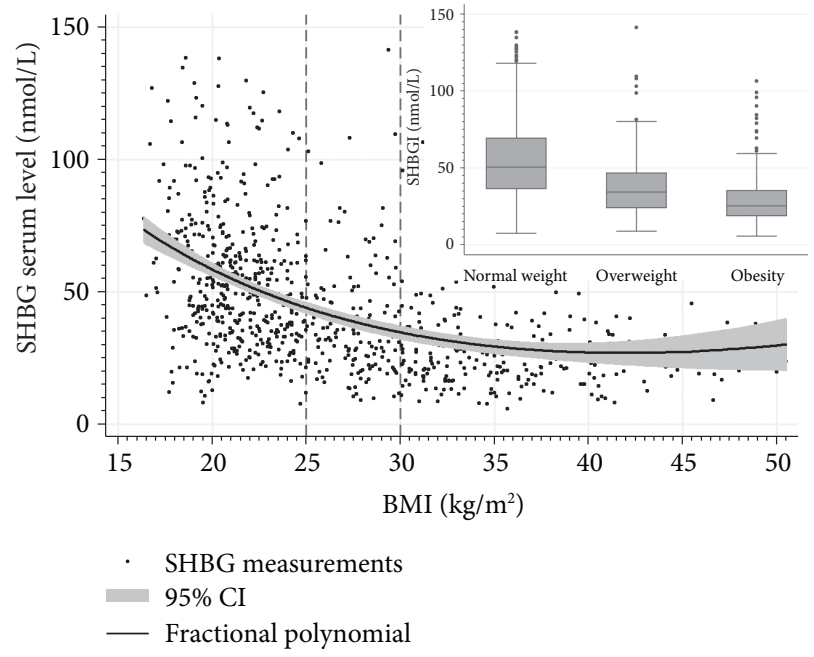

Figure 1: SHBG levels depending on nutritional status.

marker of NAFLD. Despite it, finding low SHBG level should prompt further clinical review and recommendation of standard diagnostic tests for NAFLD.

Therefore, overweight or obese women with low SHBG should be counselled with lifestyle intervention, behavioral therapy, and, in individual cases, psychotherapy and pharmacotherapy. It was indeed shown that maintaining a reduced body mass for 18 months resulted in the increase of SHBG [33].

Results of our and other studies suggest the need for a shift of the lower limit of the laboratory reference range for SHBG towards higher value. It should be taken into account 


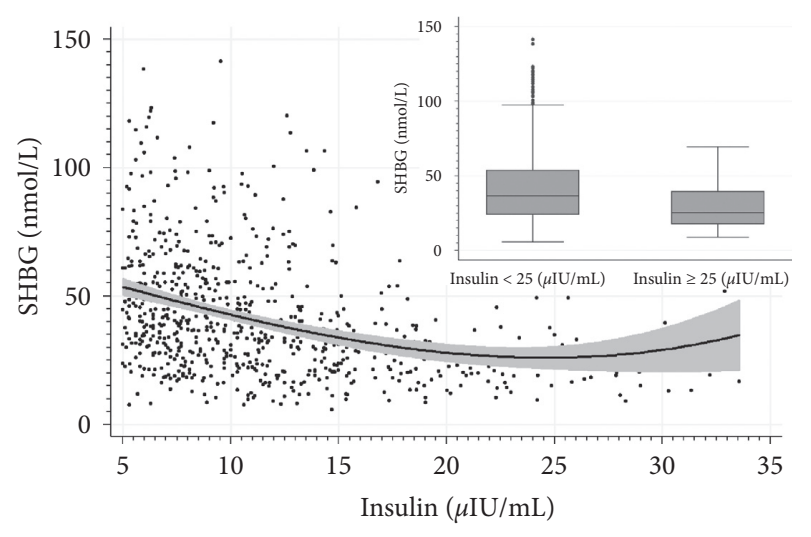

$\begin{array}{ll}\text { - } & \text { SHBG measurements } \\ \text { 95\% CI } \\ \text { - Fractional polynomial }\end{array}$

Figure 2: SHBG levels depending on insulin levels.

TABLE 3: Sensitivity, specificity, positive predictive value, negative predictive value, and accuracy of HOMA-IR > 2.0 corresponding to low circulating SHBG levels in PCOS women.

\begin{tabular}{lcc}
\hline Parameter & Value & $95 \%$ CI \\
\hline Sensitivity & $61.1 \%$ & $56.5-65.6 \%$ \\
Specificity & $71.6 \%$ & $66.9-75.8 \%$ \\
Positive predictive value & $70.7 \%$ & $65.9-75.1 \%$ \\
Negative predictive value & $62.1 \%$ & $57.6-66.5 \%$ \\
AUC & 0.711 & $0.677-0.745$ \\
\hline
\end{tabular}

AUC: area under curve; CI: confidence interval.

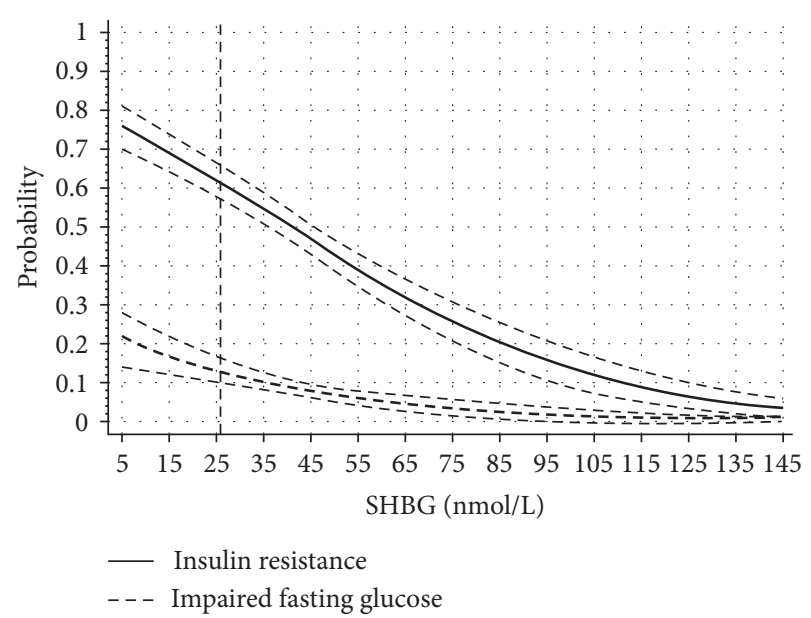

FIGURE 3: The probability of insulin resistance and impaired fasting glucose occurrence depending on SHBG levels.

that the normal ranges and units of SHBG concentration are evaluated by different methods (ECLIA, RIA, and ELISA) and may differ in the determination of the new cutoff points.

The main limitation of the present study is the lack of euglycemic-hyperinsulinemic clamp and assessment of fatty liver, as well as recognized diagnostic tests for NAFLD because NAFLD is a cause of liver insulin resistance development and decreased SHBG synthesis. It should also be noted that decreased SHBG level is an obesity-related disturbance linked to the development of liver IR, and therefore, nutritional status is one of factors indirectly affecting SHBG synthesis. Moreover, we replaced the generally accepted HOMA-IR cutoff point 2.5 for the assessment of IR in women with PCOS in a Polish population by the cutoff 2.0 calculated in our previous analysis performed in this population. On the other hand, a strength of our study is the large size of the study group and the inclusion of a homogenous group of young Caucasian women diagnosed with PCOS, with different nutritional statuses.

\section{Conclusions}

In conclusion, this is the first study estimating the probability of liver insulin resistance and impaired fasting glucose occurrence based on SHBG levels in women with PCOS. Despite the low sensitivity, the SHBG level below $42 \mathrm{nmol} / \mathrm{L}$ should result in closer monitoring for the fatty liver and prediabetes.

\section{Data Availability}

Data are available from the corresponding author on request.

\section{Conflicts of Interest}

The authors declare no conflicts of interest.

\section{Acknowledgments}

This study was funded by Medical University of Silesia, grant no. KNW-112-7/N/9/O.

\section{References}

[1] Y.-H. Lee and R. E. Pratley, "The evolving role of inflammation in obesity and the metabolic syndrome," Current Diabetes Reports, vol. 5, no. 1, pp. 70-75, 2005.

[2] E. E. Kershaw and J. S. Flier, "Adipose tissue as an endocrine organ," Journal of Clinical Endocrinology \& Metabolism, vol. 89, no. 6, pp. 2548-2556, 2004.

[3] M. Olszanecka-Glinianowicz, D. Kuglin, A. Dąbkowska-Huć, and P. Skałba, "Serum adiponectin and resistin in relation to insulin resistance and markers of hyperandrogenism in lean and obese women with polycystic ovary syndrome," European Journal of Obstetrics \& Gynecology and Reproductive Biology, vol. 154, no. 1, pp. 51-56, 2011.

[4] M. Olszanecka-Glinianowicz, P. Madej, M. Nylec et al., "Circulating apelin level in relation to nutritional status in polycystic ovary syndrome and its association with metabolic and hormonal disturbances," Clinical Endocrinology, vol. 79, no. 2, pp. 238-242, 2013.

[5] W. Rosner, D. J. Hryb, M. S. Khan, A. M. Nakhla, and N. A. Romas, "Androgens, estrogens, and second messengers," Steroids, vol. 63, no. 5-6, pp. 278-281, 1998.

[6] W. Rosner, D. J. Hryb, S. M. Kahn, A. M. Nakhla, and N. A. Romas, "Interactions of sex hormone-binding globulin with target cells," Molecular and Cellular Endocrinology, vol. 316, no. 1, pp. 79-85, 2010.

[7] F. Akin, M. Bastemir, E. Alkış, and B. Kaptanoglu, "SHBG levels correlate with insulin resistance in postmenopausal 
women," European Journal of Internal Medicine, vol. 20, no. 2, pp. 162-167, 2009.

[8] D. A. Ehrmann, R. B. Barnes, R. L. Rosenfield, M. K. Cavaghan, and J. Imperial, "Prevalence of impaired glucose tolerance and diabetes in women with polycystic ovary syndrome," Diabetes Care, vol. 22, no. 1, pp. 141-146, 1999.

[9] R. S. Legro, A. R. Kunselman, W. C. Dodson, and A. Dunaif, "Prevalence and predictors of risk for type 2 diabetes mellitus and impaired glucose tolerance in polycystic ovary syndrome: a prospective, controlled study in 254 affected Women," Journal of Clinical Endocrinology \& Metabolism, vol. 84, no. 1, pp. 165-169, 1999.

[10] Centers for Disease Control and Prevention (CDC), "Prevalence of diabetes and impaired fasting glucose in adultsUnited States, 1999-2000," Morbidity and Mortality Weekly Report, vol. 52, pp. 833-837, 2003.

[11] R. S. Legro, C. L. Gnatuk, A. R. Kunselman, and A. Dunaif, "Changes in glucose tolerance over time in women with polycystic ovary syndrome: a controlled study," Journal of Clinical Endocrinology \& Metabolism, vol. 90, no. 6, pp. 3236-3242, 2005.

[12] B. Kumarendran, M. W. O’Reilly, K. N. Manolopoulos et al., "Polycystic ovary syndrome, androgen excess, and the risk of nonalcoholic fatty liver disease in women: a longitudinal study based on a United Kingdom primary care database," PLoS Medicine, vol. 15, no. 3, Article ID e1002542, 2018.

[13] V. Jaruvongvanich, A. Sanguankeo, T. Riangwiwat, and S. Upala, "Testosterone, sex hormone-binding globulin and nonalcoholic fatty liver disease: a systematic review and metaanalysis," Annals of Hepatology, vol. 16, no. 3, pp. 382-394, 2017.

[14] H. Zhu, N. Wang, B. Han et al., "Low sex hormone-binding globulin levels associate with prediabetes in Chinese men independent of total testosterone," PLoS One, vol. 11, no. 9, Article ID e0162004, 2016.

[15] B. Fenske, H. Kische, S. Gross et al., "Endogenous androgens and sex hormone-binding globulin in women and risk of metabolic syndrome and type 2 diabetes," Journal of Clinical Endocrinology \& Metabolism, vol. 100, pp. 4595-4603, 2015.

[16] J. Hu, A. Zhang, S. Yang et al., "Combined effects of sex hormone-binding globulin and sex hormones on risk of incident type 2 diabetes," Journal of Diabetes, vol. 8, no. 4, pp. 508-515, 2016.

[17] T. Muka, J. Nano, L. Jaspers et al., “Associations of steroid sex hormones and sex hormone-binding globulin with the risk of type 2 diabetes in women: a population-based cohort study and meta-analysis," Diabetes, vol. 66, no. 3, pp. 577-586, 2017.

[18] Q. Wang, A. J. Kangas, P. Soininen et al., "Sex hormonebinding globulin associations with circulating lipids and metabolites and the risk for type 2 diabetes: observational and causal effect estimates," International Journal of Epidemiology, vol. 44, no. 2, pp. 623-637, 2015.

[19] M. W. O’Reilly, M. Glisic, B. Kumarendran et al., "Serum testosterone, sex hormone-binding globulin and sex-specific risk of incident type 2 diabetes in a retrospective primary care cohort," Clinical Endocrinology, vol. 90, pp. 145-154, 2019.

[20] T. Zhang, T. Du, W. Li, S. Yang, and W. Liang, "Sex hormonebinding globulin levels during the first trimester may predict gestational diabetes mellitus development," Biomarkers in Medicine, vol. 12, no. 3, pp. 239-244, 2018.

[21] M. M. Hedderson, F. Xu, J. A. Darbinian et al., "Prepregnancy SHBG concentrations and risk for subsequently developing gestational diabetes mellitus," Diabetes Care, vol. 37, no. 5, pp. 1296-1303, 2014.

[22] F. Mehrabian and M. Rezae, "Sex hormone binding globulin measurement before conception as a predictor of gestational diabetes in women with polycystic ovarian syndrome," Journal of Research in Medical Sciences: the Official Journal of Isfahan University of Medical Sciences, vol. 18, pp. 637-640, 2013.

[23] S. Faal, P. Abedi, S. Jahanfar et al., "Sex hormone binding globulin for prediction of gestational diabetes mellitus in preconception and pregnancy: a systematic review," Diabetes Research and Clinical Practice, vol. 152, pp. 39-52, 2019.

[24] B. Balkau and M. A. Charles, "Comment on the provisional report from the WHO consultation. European group for the study of insulin resistance (EGIR)," Diabetic Medicine: A Journal of the British Diabetic Association, vol. 16, pp. 442-443, 1999.

[25] I. R. Wallace, M. C. McKinley, P. M. Bell, and S. J. Hunter, "Sex hormone binding globulin and insulin resistance," Clinical Endocrinology, vol. 78, no. 3, pp. 321-329, 2013.

[26] P. Gyawali, S. A. Martin, L. K. Heilbronn et al., "The role of sex hormone-binding globulin (SHBG), testosterone, and other sex steroids, on the development of type 2 diabetes in a cohort of community-dwelling middle-aged to elderly men," Acta Diabetologica, vol. 55, no. 8, pp. 861-872, 2018.

[27] M. Sarkar, L. B. Van Wagner, J. G. Terry et al., "Coronary artery risk development in young adults (CARDIA) cohort (2019) sex hormone-binding globulin levels in young men are associated with nonalcoholic fatty liver disease in midlife," American Journal of Gastroenterology, vol. 114, pp. 758-763, 2019.

[28] R. Karim, F. Z. Stanczyk, R. D. Brinton, J. Rettberg, H. N. Hodis, and W. J. Mack, "Association of endogenous sex hormones with adipokines and ghrelin in postmenopausal women," Journal of Clinical Endocrinology \& Metabolism, vol. 100, no. 2, pp. 508-515, 2015.

[29] N. A. Abdella and O. A. Mojiminiyi, "Cross-sectional study of the determinants and associations of sex hormone-binding globulin concentrations in first degree relatives (FDR) of patients with Type 2 Diabetes Mellitus," Diabetes Research and Clinical Practice, vol. 133, pp. 159-167, 2017.

[30] D. Tripathy, P. Almgren, T. Tuomi, and L. Groop, "Contribution of insulin-stimulated glucose uptake and basal hepatic insulin sensitivity to surrogate measures of insulin sensitivity," Diabetes Care, vol. 27, no. 9, pp. 2204-2210, 2004.

[31] V. Jayagopal, E. S. Kilpatrick, P. E. Jennings, S. Holding, D. A. Hepburn, and S. L. Atkin, "The biological variation of sex hormone-binding globulin in type 2 diabetes," Diabetes Care, vol. 27, no. 1, pp. 278-280, 2004.

[32] S. Cassar, M. L. Misso, W. G. Hopkins, C. S. Shaw, H. J. Teede, and N. K. Stepto, "Insulin resistance in polycystic ovary syndrome: a systematic review and meta-analysis of euglycaemic-hyperinsulinaemic clamp studies," Human Reproduction, vol. 31, no. 11, pp. 2619-2631, 2016.

[33] C. Duggan, J. D. D. Tapsoba, F. Stanczyk, C.-Y. Wang, K. F. Schubert, and A. McTiernan, "Long-term weight loss maintenance, sex steroid hormones, and sex hormonebinding globulin," Menopause, vol. 26, no. 4, pp. 417-422, 2019. 